\title{
Pattern of Wnt ligand expression during chick eye development
}

\section{E. Rossi,}

F. Siwiec and C.Y.I. Yan
Departamento de Biologia Celular e do Desenvolvimento, Instituto de Ciências Biomédicas, Universidade de São Paulo, São Paulo, SP, Brasil

\section{Correspondence}

C.Y.I. Yan

Departamento de Biologia Celular

e do Desenvolvimento

ICB, USP

Av. Prof. Lineu Prestes, 1524

Sala 407

05508-900 São Paulo, SP

Brasil

Fax: +55-11-3091-7402

E-mail: ireneyan@usp.br

Research supported by FAPESP

(No. 01/09047-2).

Received October 10, 2006 Accepted May 18, 2007 ......................

\section{Abstract}

The dorsoventral axis of the eye is determined prior to optic cup invagination. A variety of signaling pathways have been implicated in the maintenance of the optic dorsoventral axis, including, but not limited to, bone morphogenetic protein 4, Sonic Hedgehog and retinoic acid. Here, we investigated the possible contribution of Wnt ligands to the establishment or maintenance of the optic axis by analyzing their expression pattern during early chick optic development. We performed in situ hybridization of Wnt-1, Wnt-3a, Wnt-4, and Wnt-5a during the optic vesicle, early optic cup and established optic cup stages and focused our analysis on the optic region. Our data showed that Wnt-5a, but none of the others, is expressed in the dorsal region of the eye starting from the Hamburger and Hamilton stage 14 (HH14). These results are supported by cryosections of the labeled optic region, which further reveal that Wnt-5a is expressed only in the dorsal retinal pigmented epithelium. Thus, we propose that Wnt-5a is a marker for dorsal retinal pigmented epithelium in chick embryos from HH14 to HH19.
The early embryogenesis of the vertebrate eye is a highly complex process where patterning is coupled with intense morphogenetic movements. The chick optic field is defined at the anterior region of the prosencephalon and develops into the bilateral optic cups during neurulation. Thereafter, these vesicles expand until they contact the overlying ectoderm, from where they will receive signals that pattern their ventral-distal region as the neural retina and the dorsalproximal region as the pigmented epithelium (reviewed in Ref. 1). Immediately after this stage, the optic vesicles invaginate, converting the ocular neuroepithelium to a bilayered optic cup. Thus, at the end of its
Key words

- Wnt-5a

- Optic vesicle

- Optic development

- Wnt

- Anterior neural tube morphogenesis, the developing eye presents clear anatomical landmarks of its axis: the lens and neural retina defining the proximaldistal axis and the ventral stalk defining the dorsoventral (DV) one.

At the molecular level, several genes are expressed and define the different ocular compartments. In the case of the DV axis, markers such as Tbx5, bone morphogenetic protein 4 (BMP4), and aldehyde dehydrogenase 1 are expressed solely in the dorsal region (2,3). In contrast, Vax, Sonic Hedgehog (Shh) and Raldh3/6 are only found in the optic cup's ventral tissues (4). The expression of these markers is determined by signaling pathways that are present in the chick 
since the Hamburger and Hamilton stage 8 (HH8) (5). Between HH8 and HH10 the optic vesicle is plastic regarding its DV axis: homochronic grafts of inverted vesicles result in DV alterations whereby the original graft conforms with the axis orientation defined by the surrounding host tissue (5). After HH10, the graft develops according to its original axial orientation. Various signaling pathways contribute to the establishment and maintenance of the DV axis. For instance, BMP4, a ligand of transforming growth factor- $\beta$ superfamily, is expressed at the dorsal optic vesicle and has been implicated in the control of the ocular DV axis. Ectopic expression of BMP4 increases the dorsal Tbx5-positive domain at the cost of the ventral Vax/Pax-2-positive domain (2). Conversely, overexpression of Ventroptin, a ventrally located BMP4 inhibitor, decreases BMP4 expression and increases Vax (3). Additional factors such as retinoic acid and Shh also play a role in optic DV patterning (1). Taken together, these reports show that optic vesicle patterning involves interactions between multiple pathways.

To identify new pathways that play a role in optic DV patterning, we investigated the expression pattern of canonical and noncanonical Wnt ligand transcripts during optic development. First, Wnt ligands of the canonical pathway and dominant-active Bcatenin can both induce expression of BMP4 and could thus play a role in maintaining dorsal optic BMP4 expression (6,7). Moreover, non-canonical Wnt have been previously identified in later stages of oculogenesis, and could participate in initial patterning events as well $(8,9)$. And finally, Wnt inhibitors have been detected in periocular regions during embryogenesis, suggesting that Wnt signaling is active in the optic region $(10,11)$.

Here we analyzed by in situ hybridization the expression of Wnt ligands in the periocular region during chick oculogenesis (12). Specifically, we focused on the expression of those Wnts that have been previously localized to the developing neural tube: Wnt1, Wnt-3a, Wnt-4, and Wnt-5a (13). The cDNAs for these genes were obtained from various laboratories (Wnt-1: Marion Wassef, CNRS, Paris, France; Wnt-3a, Wnt-4, and Wnt-5a: Tsutomu Nohno, Kawasaki Medical School, Kurashiki, Japan) and used as templates for digoxigenin-labeled cRNA probe synthesis. Since our interest was eye development, we selectively analyzed embryos ranging from optic vesicle (HH11) to late optic cup stages (HH17/18).

Briefly, whole embryos were collected, fixed with $4 \%$ paraformaldehyde, dehydrated, and re-hydrated in a methanol series. Thereafter, they were bleached with $6 \%$ $\mathrm{H}_{2} \mathrm{O}_{2}$, permeabilized with Proteinase $\mathrm{K}$ and post-fixed (4\% paraformaldehyde, $0.2 \%$ glutaraldehyde). After $2 \mathrm{~h}$ in pre-hybridization solution at $65^{\circ} \mathrm{C}(1 \mathrm{mg} / \mathrm{mL}$ Torula RNA, 100 $\mu \mathrm{g} / \mathrm{mL}$ heparin, $0.1 \%$ Tween-20, $1 \mathrm{mg} / \mathrm{mL}$ CHAPS, $10 \mathrm{mM}$ EDTA, 50\% formamide, and 1.25X SSC in Denhart's solution), the embryos were exposed overnight to the antisense cRNA probes. The negative controls were exposed to sense probes. After the hybridization step, the embryos were extensively washed and processed for immunodetection of the digoxigenin-labeled probe.

The embryos were incubated in blocking solution (2\% Roche blocking reagent, $10 \%$ sheep serum, $2 \mathrm{mM}$ Levamisole, $150 \mathrm{mM}$ $\mathrm{NaCl}, 0.1 \%$ Tween-20 in $100 \mathrm{mM}$ maleic acid buffer) for $3 \mathrm{~h}$ at room temperature. Thereafter, they were exposed overnight to anti-digoxigenin antibody conjugated to alkaline phosphatase (Roche Pharmaceuticals, Indianapolis, IN, USA). After removing excess antibody with extensive washes, labeling was identified by chromogenic processing of a substrate that resulted in a purplishblue precipitate (BM Purple; Roche). To increase the stability of the precipitate the embryos were fixed with $4 \%$ paraformaldehyde.

In embryos at the optic vesicle stage, Wnt-1 expression was more intense in the 
mesencephalic borders and midline (Figure 1A). In the posterior neural tube, Wnt-1 expression was weaker and disappeared altogether at the Hensen's node. In early optic cup stages (HH14/15, Figure 1B), Wnt-1 transcripts were detected in the dorsal region of the diencephalon and mesencephalon. In agreement with previous reports, the expression domain ended caudally at the isthmus, where it expanded ventrolaterally in the form of a ring (14). In late optic cup stages, the expression pattern of previous stages was maintained, with an increase in labeling at the borders of the metencephalon (HH16; Figure $1 \mathrm{C}$ ). We did not observe any staining in the optic vesicle or optic cup during these stages. The strong presence of Wnt-1 in the mesencephalon and at its border with the metencephalon is highly conserved amongst vertebrates (14-16). This inter-species similarity most likely is due to the high degree of conservation of Wnt-1 sequence and function. Results obtained from various model systems strongly suggest that Wnt-1 plays a relevant role in patterning both the mesencephalon and the metencephalon $(14,16)$. Mice harboring a C-terminus deletion of Wnt-1 fail to segregate mid-hindbrain junction markers and lack midbrain and metencephalic-derived structures (14). Similarly, zebrafish embryos also require Wnt-1 to maintain a proper mid-hindbrain boundary (16). Interestingly, in zebrafish, Wnt-1 and Wnt-10b act in conjunction with Wnt-3a for proper mid-hindbrain development. While Wnt-1 and Wnt-10 control mid-hindbrain compartmentalization, Wnt-3a is required for the formation of the mid-hindbrain constriction (17).

Consistent with its role in mid-hindbrain formation, our data showed that the expression pattern for Wnt-3a significantly overlapped with that of Wnt-1 during all stages analyzed. In optic vesicle-stage embryos Wnt-3a was concentrated in the mesencephalon (Figure 1D), and later on, during early optic cup stages, the Wnt-3a domain was limited caudally to the isthmus (Figure 1E). However, differently from Wnt-1, Wnt-3a displayed a ventrolateral expansion in the caudal diencephalon. There were no Wnt3a-positive optic tissues in the stages ana-
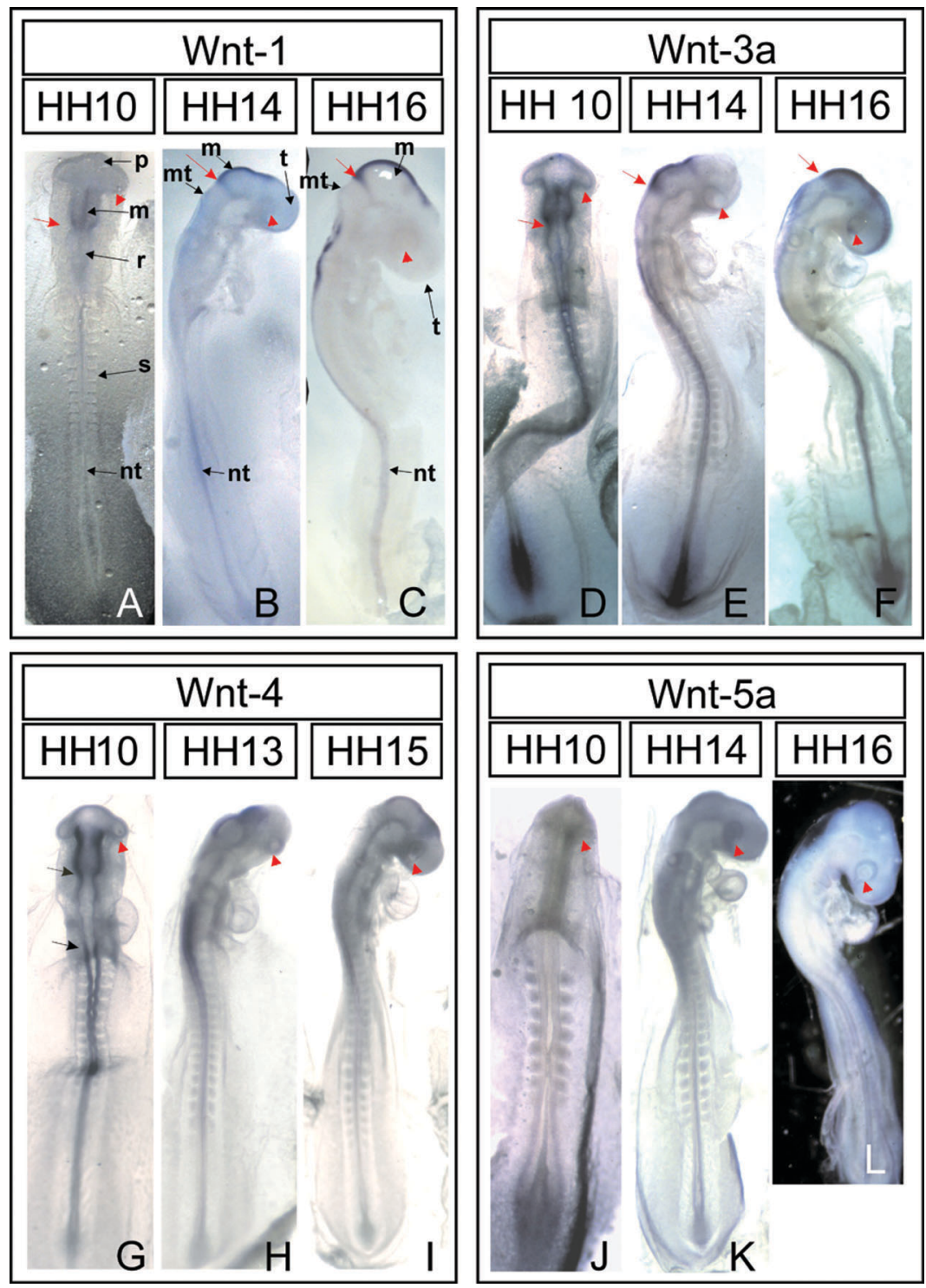

Figure 1. In situ hybridization for Wnt ligands in chick embryos during early optic development. Chick embryos in the optic vesicle (A, D, G, and J), early optic cup (B, E, H, and K) and late optic cup stages (C, F, I, and L) were hybridized with probes for Wnt-1 (A-C), Wnt3a (D-F), Wnt-4 (G-I), and Wnt-5a (J-L). Positive labeling can be identified by the accumulation of a purplish-blue precipitate. The optic vesicles and cups are indicated by the red arrowheads in all figures. In $A$, and $D$, the red arrows indicate the posterior border of the mesencephalon, which develops into the isthmus in the older embryos shown in $B, C, E$, and $F$. The anterior and posterior borders of the rhombomeres, indicated by the black arrows in $\mathrm{G}$, delimit an interruption in Wnt-4 labeling along the neural tube. $\mathrm{m}=$ mesencephalon; $\mathrm{mt}=$ metencephalon; $\mathrm{nt}=$ neural tube; $\mathrm{p}=$ prosencephalon; $r=$ rhombencephaIon; $\mathrm{s}=$ somite; $\mathrm{t}=$ telencephalon. 
lyzed here. Conservation of the overlay between Wnt-1 and Wnt-3a also occurs in various species, emphasizing the importance of their combined effect in mid-hindbrain development (13).

The Wnt-4 expression domain also overlapped with that of Wnt-1 and Wnt-3a in the mesencephalon. However, in comparison with the aforementioned ligands, the Wnt-4 expression domain in optic vesicle stages extended further into the caudal diencephalon (Figure $1 \mathrm{G}$ ) and was strikingly absent in the rhombencephalon, only to appear again in the neural tube near the first pair of so-
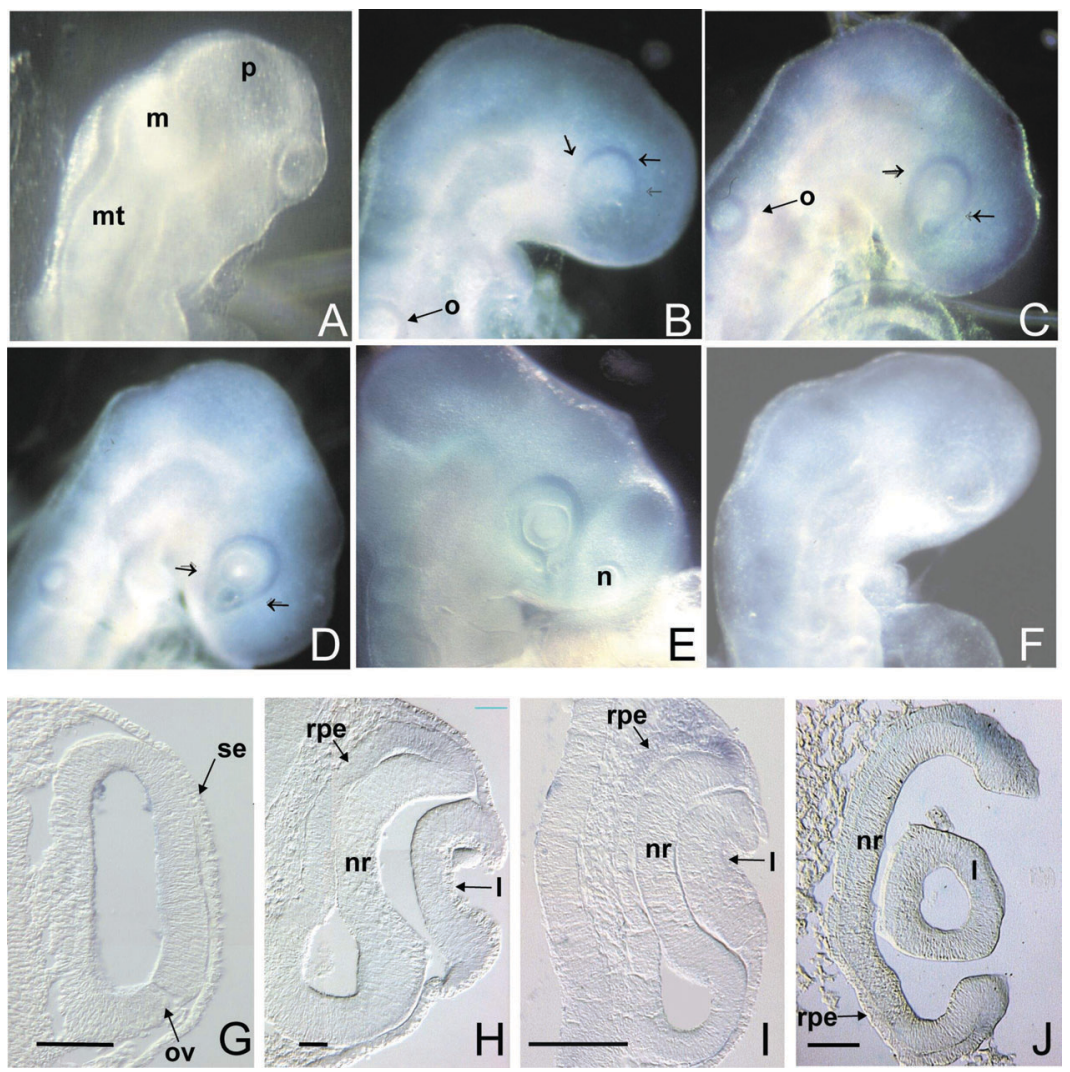

Figure 2. Wnt-5a is expressed in the dorsal retinal pigmented epithelium. Wnt-5a was not detected in $\mathrm{HH} 13$ embryos (A). However, in all stages after $\mathrm{HH} 14$, Wnt-5a expression was detected in the dorsal region of the eye by in situ hybridization of wholemount embryos at stages $\mathrm{HH}_{14}(\mathrm{~B}), \mathrm{HH} 15$ (C), $\mathrm{HH}_{16}$ (D), HH18 (E). There was no labeling in the sense controls $(F)$. Cryosection analysis of the embryos confirmed these results and showed that Wnt-5a is expressed only in the dorsal pigmented epithelium. G, HH11; H, HH14; I, HH15; J, $\mathrm{HH} 16$. Scale bars: $50 \mu \mathrm{m}$ in $\mathrm{G}$ and $\mathrm{H} ; 90 \mu \mathrm{m}$ in I; $100 \mu \mathrm{m}$ in J. Arrows denote the ventralmost limit of the Wnt-5a expression domain. In all figures, the dorsal region is closer to the top. $I=$ lens; $\mathrm{m}=$ mesencephalon; $\mathrm{mt}=$ metencephalon; $\mathrm{n}=$ nasal pit; $\mathrm{nr}=$ neuroretina; $\mathrm{o}=$ otic vesicle; ov = optic vesicle; $p=$ prosencephalon; $r p e=$ retinal pigmented epithelium; $s e=$ surface ectoderm. mites (Figure 1G). This absence from the rhombencephalon has been reported previously to be a particular feature of chick embryos $(13,14)$. In early optic cup-stage embryos, the labeling of the posterior neural tube persisted, while there was a ventrolateral increase in the Wnt-4 expression domain in the diencephalon (Figure $1 \mathrm{H}$ ). The diencephalic domain took the shape of a chevron, and remained so in later stages as well. In the mesencephalon, the caudal limit of Wnt-4 was in the isthmus and, similarly to Wnt-1, it took the form of a ventrolaterally expanded ring. This expression pattern persisted in later stages. There was no expression of Wnt-4 in ocular tissues during any of the stages analyzed here.

Wnt-5a expression in optic vesicle stages was restricted to Hensen's node and did not appear in the neural tube, confirming previous reports of this gene's expression pattern (Figure 1J-L; 13). The first appearance of Wnt-5a in the neural tube is in the optic cup, in stage HH14 embryos (Figures 1K, 2B). Thereafter, it remains localized to the dorsal region of the eye until $\mathrm{HH} 18$, the latest stage we analyzed (Figure 2C-E). The earliest detection of Wnt-5a at stage HH14 coincides with the first stage when most DV markers are clearly compartmentalized. For instance, Tbx5 is expressed throughout the optic vesicle until stage HH14, after which it is restricted to the dorsal retina and retinal pigmented epithelium (2). Similarly, Ventroptin and Vax are only restricted to the ventral region after stage HH14 (3).

For detailed analysis of the expression pattern, the embryos were cryoprotected with a $20 \%$ sucrose solution, embedded in OCT (Tissue-Tek) and sliced in a cryostat. The cryosections confirmed that the earliest appearance of Wnt-5a in the eye was at HH14 (compare Figure 2G with 2H). Furthermore, this early expression was restricted to the dorsal retinal pigmented epithelium (RPE) and was absent from the developing retina. This restriction was maintained throughout 
all the stages analyzed here. At later stages, others have reported that the Wnt-5a domain expands to the remainder of the RPE and is also found in the equatorial region of the lens $(8,9)$. On the basis of the present data, we propose Wnt-5a as an early dorsal RPE marker for chick embryos between HH14 and HH19.

The restriction of $\mathrm{Wnt}-5 \mathrm{a}$ to the dorsal RPE in HH14 embryos is particularly interesting when compared to previously identified RPE marker genes and argues for an underlying DV axis in the RPE. The best characterized RPE marker thus far is the microphtalmia transcription factor (MITF). MITF expression precedes that of Wnt-5a. Its appearance initiates the expression of key genes that define the differentiation of the RPE. Consistent with its role, in the optic cup, MITF is found homogeneously throughout the developing RPE (18).

However, evidence suggests that the RPE has distinct DV compartments during its development. First, cell proliferation is main- tained for longer periods of time in the ventral RPE (19). Furthermore, in MITF-mutant mice, only the dorsal RPE is converted to retina (18). Finally, we show here that Wnt$5 \mathrm{a}$ is initially expressed in the dorsal RPE. Similarly, Wnt-2b is also expressed in the dorsal RPE during HH14 (20). Taken together, these data suggest not only that the RPE has a DV axis during development, but also that both the canonical and non-canonical Wnt pathways could play a role in early patterning of the RPE.

\section{Acknowledgments}

The authors would like to thank Marion Wassef (CNRS, Paris, France) and Tsutomu Nohno (Kawasaki Medical School, Kurashiki, Japan) for generously sharing their plasmids. We would also like to acknowledge Tatiana Hochgreb and Ricardo Borges for their help in optimizing the in situ hybridization and cryosection protocols.

\section{References}

1. Chow RL, Lang RA. Early eye development in vertebrates. Annu Rev Cell Dev Biol 2001; 17: 255-296.

2. Koshiba-Takeuchi K, Takeuchi JK, Matsumoto K, Momose T, Uno $\mathrm{K}$, Hoepker V, et al. Tbx5 and the retinotectum projection. Science 2000; 287: 134-137.

3. Sakuta $H$, Suzuki R, Takahashi $H$, Kato A, Shintani T, lemura S, et al. Ventroptin: a BMP-4 antagonist expressed in a double-gradient pattern in the retina. Science 2001; 293: 111-115.

4. Sasagawa S, Takabatake T, Takabatake Y, Muramatsu T, Takeshima K. Axes establishment during eye morphogenesis in Xenopus by coordinate and antagonistic actions of BMP4, Shh, and RA. Genesis 2002; 33: 86-96.

5. Uemonsa T, Sakagami K, Yasuda K, Araki M. Development of dorsal-ventral polarity in the optic vesicle and its presumptive role in eye morphogenesis as shown by embryonic transplantation and in ovo explant culturing. Dev Biol 2002; 248: 319-330.

6. Haegele L, Ingold B, Naumann H, Tabatabai G, Ledermann B, Brandner S. Wnt signalling inhibits neural differentiation of embryonic stem cells by controlling bone morphogenetic protein expression. Mol Cell Neurosci 2003; 24: 696-708.

7. Kim JS, Crooks H, Dracheva T, Nishanian TG, Singh B, Jen J, et al. Oncogenic beta-catenin is required for bone morphogenetic protein 4 expression in human cancer cells. Cancer Res 2002; 62: 2744-
2748.

8. Jin EJ, Burrus LW, Erickson CA. The expression patterns of Wnts and their antagonists during avian eye development. Mech Dev 2002; 116: 173-176.

9. Fokina VM, Frolova El. Expression patterns of Wnt genes during development of an anterior part of the chicken eye. Dev Dyn 2006; 235: 496-505.

10. Terry K, Magan H, Baranski M, Burrus LW. Sfrp-1 and Sfrp-2 are expressed in overlapping and distinct domains during chick development. Mech Dev 2000; 97: 177-182.

11. Ladher RK, Church VL, Allen S, Robson L, Abdelfattah A, Brown NA, et al. Cloning and expression of the Wnt antagonists Sfrp-2 and Frzb during chick development. Dev Biol 2000; 218: 183-198.

12. Stern $C D$. Detection of multiple gene products simultaneously by in situ hybridization and immunohistochemistry in whole mounts of avian embryos. Curr Top Dev Biol 1998; 36: 223-243.

13. Hollyday M, McMahon JA, McMahon AP. Wnt expression patterns in chick embryo nervous system. Mech Dev 1995; 52: 9-25.

14. Bally-Cuif L, Cholley B, Wassef M. Involvement of Wnt-1 in the formation of the mes/metencephalic boundary. Mech Dev 1995; 53 : 23-34.

15. Wilkinson DG, Bailes JA, McMahon AP. Expression of the protooncogene int-1 is restricted to specific neural cells in the developing 
mouse embryo. Cell 1987; 50: 79-88.

16. Lekven AC, Buckles GR, Kostakis N, Moon RT. Wnt1 and wnt10b function redundantly at the zebrafish midbrain-hindbrain boundary. Dev Biol 2003; 254: 172-187.

17. Buckles GR, Thorpe CJ, Ramel MC, Lekven AC. Combinatorial Wnt control of zebrafish midbrain-hindbrain boundary formation. Mech Dev 2004; 121: 437-447.

18. Bumsted KM, Barnstable CJ. Dorsal retinal pigment epithelium dif- ferentiates as neural retina in the microphthalmia $(\mathrm{mi} / \mathrm{mi})$ mouse. Invest Ophthalmol Vis Sci 2000; 41: 903-908.

19. Lee CS, May NR, Fan CM. Transdifferentiation of the ventral retinal pigmented epithelium to neural retina in the growth arrest specific gene 1 mutant. Dev Biol 2001; 236: 17-29.

20. Cho SH, Cepko CL. Wnt2b/beta-catenin-mediated canonical Wnt signaling determines the peripheral fates of the chick eye. Development 2006; 133: 3167-3177. 